\title{
Resource Management in Civil Construction Using RFID Technologies
}

\author{
Changyoon $\mathrm{Kim}^{1}$, Yeonjong $\mathrm{Ju}^{2}$, Hyoungkwan $\mathrm{Kim}^{3}$ and Jung-Hoon $\mathrm{Kim}^{4}$ \\ ${ }^{1}$ School of Civil and Environmental Engineering, College of Engineering, Yonsei University, 134 Shinchon- \\ dong, Seodaemun-gu, Seoul 120-749, Republic of Korea ; PH (+822) 2123-7940 ; FAX (+822) 364-5300 ; e- \\ mail: changyoonkim@yonsei.ac.kr \\ ${ }^{2}$ School of Civil and Environmental Engineering, College of Engineering, Yonsei University, 134 Shinchon- \\ dong, Seodaemun-gu, Seoul 120-749, Republic of Korea ; PH (+822) 2123-7940 ; FAX (+822) 364-5300 ; e- \\ mail: j970132@hotmail.com \\ ${ }^{3}$ Assistant Professor, School of Civil and Environmental Engineering, College of Engineering, Yonsei \\ University, 134 Shinchon-dong, Seodaemun-gu, Seoul 120-749, Republic of Korea ; PH (+822) 2123-5799; \\ FAX (+822) 364-5300 ; e-mail: hyoungkwan@yonsei.ac.kr \\ ${ }^{4}$ Assistant Professor, School of Civil and Environmental Engineering, College of Engineering, Yonsei \\ University, 134 Shinchon-dong, Seodaemun-gu, Seoul 120-749, Republic of Korea ; PH (+822) 2123-5804; \\ FAX (+822) 364-5300 ; e-mail: junghoon@yonsei.ac.kr
}

\begin{abstract}
Large amount of construction resources and their scattered locations in a civil construction site make it extremely difficult for project managers to effectively utilize the resources. In order to manage construction resource effectively, a Radio Frequency Identification (RFID) technology was used in this research. RFID tags for metallic objects were attached to structural components used in a cable-stayed bridge. The RFID reader and antennas were installed at the construction site for tracking the structural components and the acquired data were transferred to the head office via Local Area Network (LAN). The proposed RFID system enabled real-time monitoring of the construction materials in the civil construction site.
\end{abstract}

\section{Introduction}

Large amount of construction resources and their scattered locations in a civil construction site make it extremely difficult for project managers to effectively utilize the resources. For this reason, there are strong needs for managing construction resources in a real-time manner (Kim et al., 2008). Diverse sensing techniques such as Radio Frequency Identification (RFID) technology and flash Laser Radar (LADAR) were being used in construction sites (Song et al. 2006; Kim et al. 2008). The RFID technology is a wireless object identification method relying on radio frequency signals. A RFID reader transmits the signal through the Transmission (TX) antenna and receives it which is reflected by the RFID tag through the Reception (RX) antenna. In this research, a preliminary experiment was conducted to characterize a RFID system composed of readers and tags. Capability of the reader and tags for metal and non-metal objects were identified using the frequency of $910.4 \sim 913.6 \mathrm{Mhz}$ UHF (Ultra High Frequency), and off the shelf tags classified as Electronic Product Code (EPC) class 1, Generation 2 (GEN II) RFID. Finally, a case study was conducted to confirm the applicability and robustness of the RFID system in a real civil construction site.

\section{Research Background}

Pioneering researchers have tried to use RFID technologies for the contactless identification of construction resources in various construction sites, including plant and building construction. However, RFID application in civil engineering works such as bridge construction has not yet been reported. Song et al. (2006) suggested a strategy for tracking locations of construction materials using the RFID technology. In their study a construction vehicle was first located, and By using Global Positioning System (GPS), RFID, and Bluetooth communication protocol (IEEE 802.15.1), construction vehicles were automatically tracked. The RFID technology is often used for facility management and maintenance. Ergen et al. (2007) presented a RFID application strategy for management of fire valves. In order to record inspection history of fire 
valves, RFID tags were used. Ko (2008) developed a web-based facility management information system, along with the RFID technology, to manage and maintain building facility. This is a trend found in other research efforts, too. Through the combination of RFID and 4D CAD (Computer Aided Design), construction progresses can automatically be monitored and visualized (Chin et al., 2008).

\section{Preliminary Experiment}

The drawback of the normal RFID system is that the tags are not easily detected in a metallic environment (Kim et al. 2008). The radio frequency signal is interfered by metallic materials so that RFID tags cannot reflect the signal that transmitted from the TX antenna. This brings about serious problem because the cable stayed bridge, chosen as a test case for RFID applicability, consists of many metallic structural objects.

To overcome this shortcoming, metal tags, which were specially designed for detecting metal objects, were used. The metal tag is composed of RFID chip, antenna, and an insulator. Insulators create an extra layer between the RFID tag (chip and antenna) and the object that construction managers want to identify. This is to satisfy the condition where the radio frequency signal is not interfered by the metallic environment.

To measure the readability of RFID tags, the number of tag detection during a specified time duration (1 min) was counted. In addition, the reading ranges (distance) and angles were also measured. The experiment of reading ranges and angles was repeated three times and the average value was used for the analysis. Fig. 1(a) shows the experiment results for non-metal tags. When the traditional RFID tag was placed in front of the reader (with the angle of $0^{\circ}$ ) in a non-metallic environment, the maximum reading range was seven meters from the RFID reader. When the traditional RFID tag was placed on the oblique line (with the angles of Left (L) $45^{\circ}$ and Right (R) $45^{\circ}$ ), the maximum reading range was four meters and five meters, respectively. The reading ranges of the tag were only one meter and two meters at the angles of $\mathrm{R} 90^{\circ}$ and $\mathrm{L} 90^{\circ}$, respectively.

A metal RFID tag was also used in this experiment. The reading range of the tag was almost same as the traditional RFID tag when it comes to non-metallic objects. However, the result was quite different for metallic objects. Fig. 2 shows the experimental results of reading ranges and angles of the metal tag. None was identified when the traditional RFID tag was attached to the metallic materials. However, the RFID tag for metallic environment was well identified even when the tag was attached to the metallic materials. The level of reading frequency was a little decreased, but the RFID tag was successfully identified by the reader.

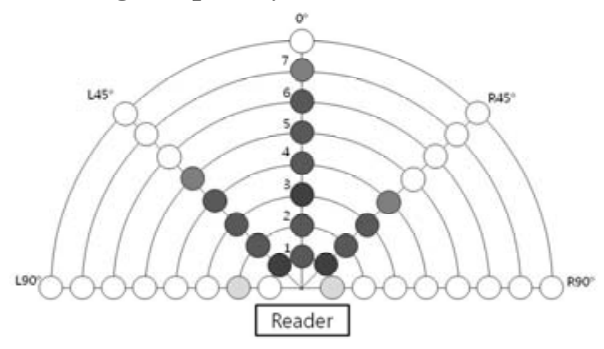

(a) Non-metal Tag

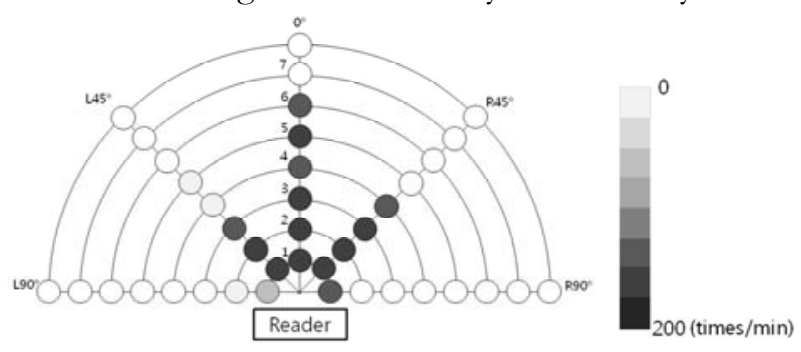

(a) Metal Tag

Fig. 1. Reading ranges and angles (non-metallic environment)

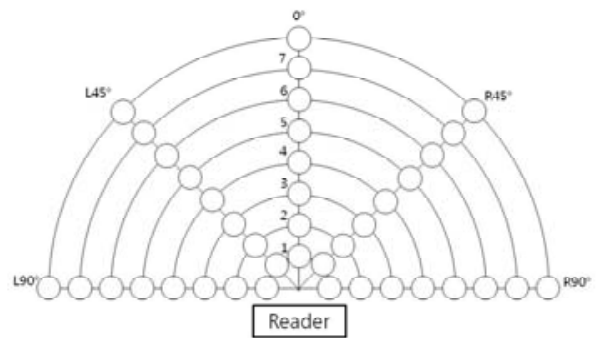

(a) Non-metal Tag

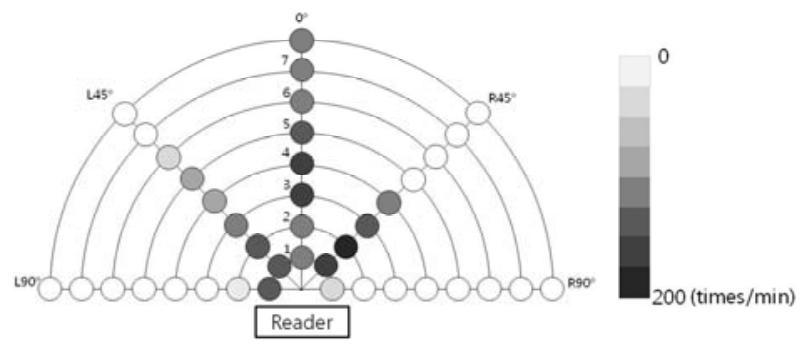

(a) Metal Tag

Fig. 2. Reading ranges and angles (metallic environment) 


\section{Case Study and System Architecture}

A case study is being conducted in a cable-stayed bridge construction site in Chungcheong-bukdo, Jecheon, Korea. Precast concrete panels that will comprise the upper deck of the bridge segment was chosen as the material type to be monitored (Fig. 3). The panels are being fabricated in a lot approximately $50 \mathrm{~m}$ away from the main entrance of the construction site. Since the panels consists of cement, aggregates, and reinforcing bars which are metal, the metal RFID tags are used for successful identification. RFID tags that were purchased were first tested in an indoor environment before being deployed in the site. 19 RFID tags out of 197 (9.64\%) turned out to be defective, and the defective tags are not used for the case study. Since the concrete panels are still being fabricated, not all the non-defective tags are deployed yet. The RFID technology-based material tracking system is now in place and the initial tracking result is coming out.

To facilitate successful material management, Radio Frequency Identification (RFID) and Local Area Network (LAN) are used in this study (Fig. 4). To identify construction materials with RFID tags at the construction site, two pairs of antennas (two TXs and two RXs) and a RFID reader were installed at the entrance of the construction site. Two pairs of antennas (TX and RX antenna) are installed to broaden the reading range of RFID readers. When the material with an RFID tag enters into the detection zone of the reader, it can be identified and the information stored in the tags can be transferred to the main database server via LAN. A preliminary database was developed using MS-Access and the VDSL (very high-data rate digital subscriber line) is used for the internet connection.

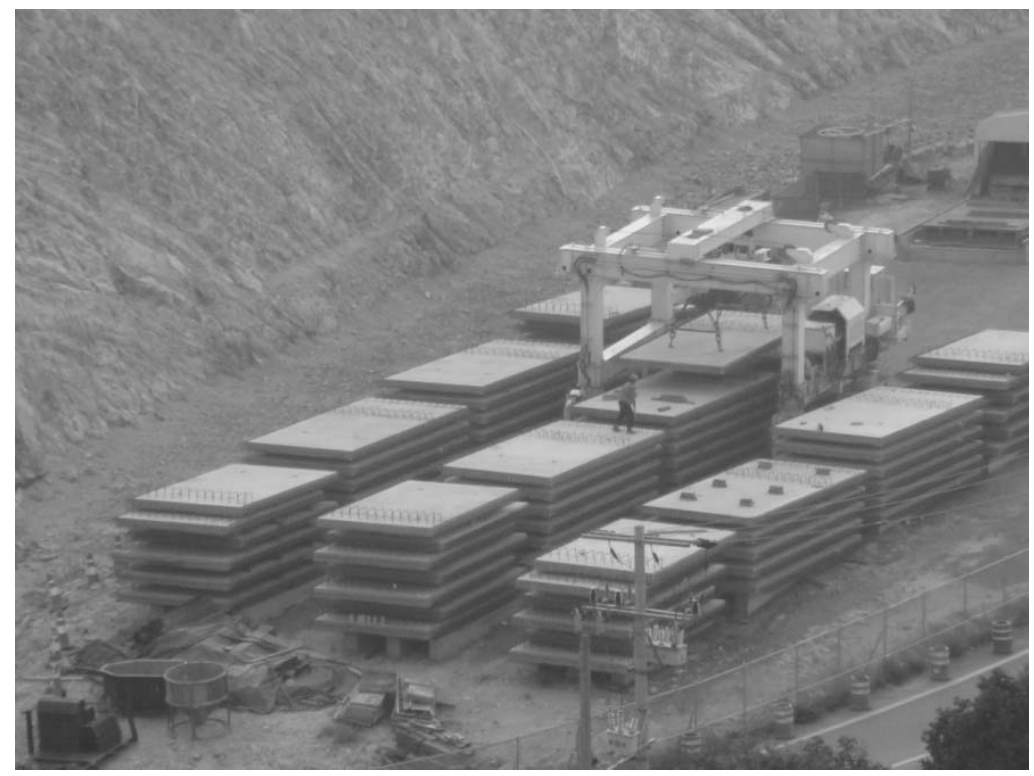

Fig. 3. Precast concrete panels

\section{Conclusions}

This research presents an effort to evaluate the applicability of the RFID technology in managing resources in civil construction sites. Coupled with the LAN connection, the system is able to provide the management with real time material tracking information in any place where the internet connection is available, including construction site office and head office. The special tags for a metallic environment were also adopted to enhance the robustness of the RFID systems in the cable-stayed bridge construction site where diverse construction materials were used.

This study is unique and contributory to the existing body of knowledge in the sense that to the knowledge of the authors, this is the first trial where RFID technology is used in a civil construction. Although RFID technologies have shown big potentials in architectural engineering or plant constructions, no significant effort has yet been reported with regard to the RFID application in civil construction sites. Future study will focus on extracting statistically meaningful and reliable conclusions as to how best to be able to utilize RFID technologies in civil engineering construction such as a cable stayed bridge. 


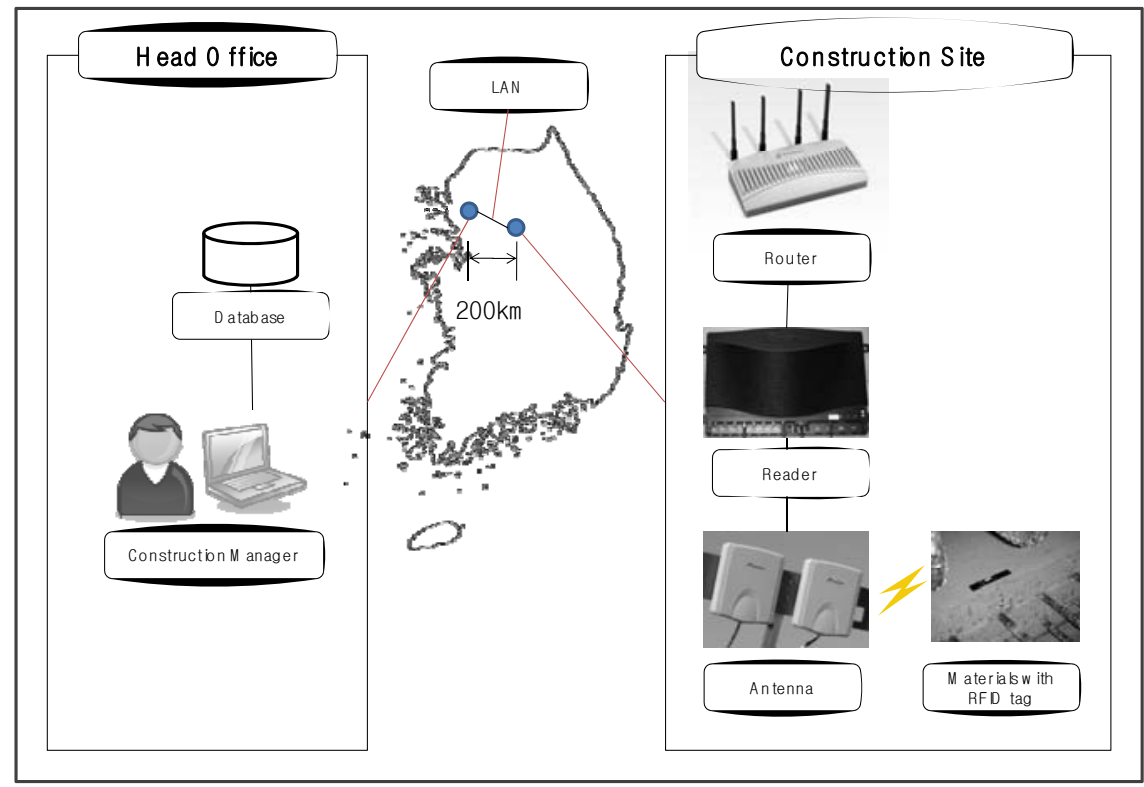

Fig. 4. Resource management system architecture

\section{Acknowledgement}

This work was supported by a Grant (KRF-2008-331-D00611) from the Korea Research Foundation and a Grant (07UrbanRenessanceB03) from High-Tech Urban Development Program funded by the Korean Ministry of Land, Transport, and Maritime Affairs.

\section{References}

[1] Chin, S., Yoon, S., Choi, C., and Cho, C. (2008). "RFID + 4D CAD for Progress Management of Structural Steel Works in High-Rise Buildings.” Journal of Computing in Civil Engineering, 22(2), 7489.

[2] Eegen, E., Akinci, B., East, B., and Kirby, J. (2007). "Tracking Components and Maintenance History within a Facility Utilizing Radio Frequency Identification Technology.” Journal of Computing in Civil Engineering, 21(1), pp. 11-20.

[3] Kim, C., Kim, H., Ryu, J., Kim, C., Kim, M. K. (2008). "Ubiquitous Sensor Network for Real-time Construction Material Monitoring." Proceedings of International Conference on Computing in Civil and Building Engineering (ICCCBE 2008), Tsinghua University, Beijing, China.

[4] Kim, C., Kim, H., Han, S. H., Kim, C., Kim, M. K., and Park, S. H. (2008). "Developing a Technology Roadmap for Construction R\&D through Interdisciplinary Research Efforts." Automation in Construction, In press.

[5] Kim, C., Son, H., Kim, H., and Han, S. H. (2008). "Applicability of Flash LADAR to 3D Spatial Information Acquisition and Modeling on a Construction Site." Canadian Journal of Civil Engineering, 35, 1131-1341.

[6] Ko, C.-H. (2008). "RFID-based Building Maintenance System.” Automation in Construction, In press.

[7] Lu, M., Chen, W., Shen, X., and Lam, H.-C., and Liu, J. (2007). "Positioning and Tracking Construction Vehicles in Highly Dense Urban Areas and Building Construction Sites." Automation in Construction, 16(5), 647-656.

[8] Song, J., Haas, C. T., and Caldas, C. H. (2006). "Tracking the Location of Materials on Construction Job Sites.” Journal of Construction Engineering and Management, 132(9), 911-918. 